\title{
Derrick Henry Lehmer
}

\author{
by \\ John Brillhart (Tucson, Ariz.)
}

Derrick Henry Lehmer (DHL) began life in Berkeley, California on February 23, 1905. He was one of the five children of Derrick Norman Lehmer (DNL) and [Clara] Eunice Mitchell. As he was growing up, he was greatly influenced by his father who was a professor of mathematics at the University of California at Berkeley. He learned about computing in mathematics and the making of sieves from his father, who had extensively used manual sieves in producing his two famous books [1] and [2]. His pre-university education was in the Berkeley public school system.

As an undergraduate at U. C., Berkeley, DHL adapted an idea of his father for making a sieving mechanism with paper strips, rubber bands, and a sheet of plywood. In his re-design he replaced the paper strips by bicycle chains hanging on sprockets attached to a shaft (with a counter) that was turned by an electric motor. The machine ran without attention, stopping only when a solution was found. (See paper \#9. A list of the publications of DHL is given following this article.) With the aid of this rapid parallel "anding" machine, as well as the other sieves that he and his associates designed and built throughout his lifetime, he solved many problems in number theory that were often far beyond what anyone else could solve, such as the factoring and primality testing of large integers and the discovery of pseudo-squares. The original bicycle-chain sieve no longer exists, though the Computer Museum in Boston, Mass. has a working replica of this machine, as well as the photo-electric number sieve, and two other sieves which were donated to the museum by DHL. For more information about these machines see \#165, pp. xxix-xlii, \#155, [4], and [5].

Also, while he was an undergraduate, he met a young student, Emma Markovna Trotskaia (born in 1906 in Samara, Russia), who had come to U. C., Berkeley in 1924 from Harbin to study engineering. After a couple of years of undergraduate study, Emma changed to mathematics and began to assist DNL and DHL in their work (see \#7), such as the making of stencils for factoring integers [3]. In 1927 DHL received his bachelor's degree in 
physics from Berkeley, whereupon he went to the University of Chicago for graduate studies with L. E. Dickson.

In 1928 Emma received her bachelor's degree in mathematics at Berkeley and the two were married. Since DHL didn't like working under Dickson and since Brown University offered him an instructorship, the couple moved to Providence, Rhode Island. In his first year at Brown, DHL completed a master's degree and in 1930 he finished his $\mathrm{PhD}$ (paper \#16) under Tamarkin, who, though he was not a number theorist, had a broad understanding of mathematics.

From 1930 to 1932 DHL was a National Research Fellow at Cal. Tech. and Stanford. In 1933 the photo-electric sieve became operational and was displayed (non-operationally) that summer at the World's Fair in Chicago. In the fall the Lehmers went to the Institute for Advanced Studies at Princeton under another fellowship. The following year DHL obtained a more permanent position at Lehigh University where he stayed until 1940, except for a year's leave in 1938-39 to Cambridge, England on a Guggenheim Fellowship, during which time the Lehmers met Hardy, Littlewood, and the engaging computational mathematician J. C. P. Miller at Cambridge and Mordell, Davenport, Mahler, and Erdős at Manchester. Following this respite, they returned to Lehigh with their two young children on almost the last ship to the United States before the Nazi submarine menace began in the Atlantic.

In 1940, the Lehmers were able to leave Bethlemen, PA to accept a proffered position in the mathematics department at Berkeley, a position DHL held with a few interruptions until he became an emeritus professor in 1972. In the two decades that followed his retirement, the Lehmers continued their mathematical research, as always, with a profound admiration and devotion for each other. DHL passed away quietly in Berkeley on May 22, 1991.

In 1945-46 DHL went to Aberdeen Proving Ground to assist in setting up and running the ENIAC, the first automatic computer in the United States. In 1950, when the loyalty oath uproar occurred at Berkeley, he went to UCLA for two years to head the Bureau of Standards' Institute for Numerical Analysis, where the early computer, the SWAC, was running. He returned to Berkeley when the loyalty oath was declared unconstitutional by the courts.

As a lecturer DHL was much appreciated not only for his classical scholarship in mathematics and number theory, but also for his dry sense of humor and his wit. (In his number theory classes during the period when the U.S. refused to recognize mainland China, he always referred to the Chinese Remainder Theorem as the Taiwan Remainder Theorem.) He was a down-to-earth man who eschewed abstraction for its own sake. He loved and valued formulas and numerically examined the mathematical questions 
that interested him. He always took a deeply computational or constructive approach to mathematics. It is worth quoting him on this (see paper \#108, p. 224):

"When I was young, mathematicians still had a slight feeling of guilt about a proof that establishes only the existence of a solution of a problem, without supplying a constructive procedure for finding the solution. Many times I was told that the existence proof was only the first step and, now that the solution is known to exist, very soon some, presumably less pure, mathematician would come up with a constructive solution. Meanwhile, the thing to do is to generalize the existence proof to Banach space and beyond. In almost all cases, I am still waiting for the second man. Often I am asked the rhetorical question 'How can you even begin to solve a problem until it is known that the solution exists?' The answer to this smug question is 'easily! I have done it many times'."

The breadth of Lehmer's mathematical work is best judged by the 17 subject headings he chose for the 1981 publication of his Selected Papers (for details see below). These headings are: Lucas' Functions, Tests for Primality, Continued Fractions, Bernoulli Numbers and Polynomials, Diophantine Equations, Numerical Functions, Matrices, Power Residues, Analytic Number Theory, Partitions, Modular Forms, Cyclotomy, Combinatorics, Sieves, Equation Solving, Computing Techniques, and Miscellaneous. In some of these areas his results are very well known. For example, under Lucas' functions there is his influential thesis (\#16) which extended Lucas' work on the divisibility properties of what are called "Lucas sequences" to a special type of fourth order sequence. The terms in these more general sequences are now called "Lehmer numbers" [6].

DHL was also interested in primality testing throughout his life. He clarified and extended Lucas' use of the Fermat congruence in primality testing, making its use well known. Paper \#143 with John Selfridge and this writer carried this topic a great deal further. DHL is perhaps best known for his sharp and definitive form of Lucas' primality test (\#38) for the Mersenne number $M_{p}, p$ a prime: Let $S_{n+1}=S_{n}^{2}-2, S_{1}=4$. Then $M_{p}$ is a prime if and only if $M_{p}$ divides $S_{p-1}$.

DHL was involved throughout his life with the theory and practice of factoring integers. Besides the many impressive factorizations he obtained while working by hand or with his sieves, he also published the important paper with Powers (\#25) in which they present a new factoring algorithm. Although the attitude in the paper is negative toward the algorithm as a hand method, this was the paper that precedes the development of the continued fraction method by M. Morrison and this writer. It was this method which was powerful enough on a computer to factor the seventh Fermat number in 1970 in 45 minutes. 
One matter of particular interest is his work on the density of primes having a given primitive root $g$. Artin had conjectured that this density would be the same (about 3/8) regardless of what the value of $g$ was. In \#103 data are presented that seem to contradict this conjecture, at least for small primes such as $-3,5$, and -7 , for which the set of primes having one of them as a primitive root has a much larger density than the average. Lehmer's observation was later shown to follow from the Generalized Riemann Hypothesis by C. Hooley. In \#104 DHL discusses in the same heuristic fashion the densities of prime values of quadratic polynomials that have a certain $g$ as a primitive root. In this case he presents examples which have quite high densities.

The work of DHL on the partition function $p(n)$ is outstanding and is recognized as such. The first of his 5 papers on this subject (\#41) dealt with verifying two cases of Ramanujan's conjecture, viz. that $5^{4}$ divides $p(599)$ and $11^{3}$ divides $p(721)$. These values were computed using the Hardy-Ramanujan series, and they verified the conjectures. However, it was not known that this series converges. In \#46 DHL showed that the series actually diverges, but can nonetheless be reliably used to compute values of $p(n)$, because after a determinable number of terms the partial sums differ from the actual value by less than $1 / 2$. His later papers in the series \#47, \#48, and \#56 all deal in an elegant way with the question of estimating the remainder term in the series. He also settles two more divisibility questions about $p(n)$ by computing values using the divergent series.

Another major study concerned the roots of the Riemann zeta function. He was the first person to make an extensive calculation of these roots on an automatic computer (\#83). He found that the first 10000 zeros of the zeta function lie on the critical line. In \#87 he extended this calculation and showed that the first 25000 zeros are on the critical line. In both these calculations he discusses statistics relative to the Gram intervals and points out a "near miss" for the Riemann hypothesis. These two papers were also written to show how the zeros of the zeta function can be effectively computed from a programming point of view.

In addition to the influential mathematical work mentioned above, there are two well-known conjectures due to Lehmer that have yet to be settled: (1) (\#69) The Ramanujan tau function never vanishes; (2) (\#32) If $\phi(n)$ divides $n-1$, then $n$ is prime. An interesting question he also asked (\#34), to which he may well have provided the final answer, is whether for any $\varepsilon>0$ there exists a monic polynomial with integer coefficients for which the absolute value of the product of its zeros that lie outside the unit circle is less than $1+\varepsilon$. This problem has come to be known in the theory of Diophantine approximations as the "Lehmer problem". It seems that the 
answer is negative when $1+\varepsilon$ equals the relevant product for his polynomial: $x^{10}+x^{9}-x^{7}-x^{6}-x^{5}-x^{4}-x^{3}+x+1$.

The algorithms he devised were not "Gedanken" algorithms, but practical algorithms he used to get information about what he was studying at the time. His papers on computing, which usually contain material dealing with his current research, abound in the ideas, methods, insights, and discoveries of a man who feels that computing in its various forms, and the theories that go with it, should be primarily practical. To have an eminent professor on the faculty with this point of view was particularly encouraging to students of a similar frame of mind (like myself) who were trying to survive in the ever-growing, rarified atmosphere of abstraction that was so common in Berkeley during his years there.

As a thinker, DHL was sagaciously independent, not being devoted to dogmas, systems, or rituals. On the other hand, he comfortably cooperated with others but strongly disliked slavery in any form, especially in the professor-student relationship. Many of his graduate students worked by themselves, conferring with DHL only on occasion to show him their progress.

In the some sixty years during which they collaborated, the Lehmers were a research team who personally influenced a large number of people with their knowledge, their courtesy and sociability, and their fine mathematical work. There is little doubt that one of their most enduring contributions to the world of mathematicians is their founding of the West Coast Number Theory Meeting in 1969. This meeting, which has been held in the western United States in December of every year since that time, has provided an informal and often merry environment in which old friends can meet and younger mathematicians can present their work. This meeting will remain a lasting tribute to the Lehmers.

Throughout his life DHL was a very well known number theorist and computational mathematician, whose papers contain fundamental contributions to many parts of mathematics. His published books are items \#61 and \#165. In 1943 he became one of the first editors of the new journal MTAC (later re-named Mathematics of Computation), a position he held until 1954. In 1975 the editors of that journal honored him on his 70th birthday by dedicating a special issue to him. In 1958 DHL began his long tenure as a member of the Advisory Board of Acta Arithmetica. In 1981 three volumes of his papers were published by The Charles Babbage Research Centre. These volumes, titled Selected Papers of D. H. Lehmer, are still available in hard or soft cover from that centre at P.O. Box 272, St. Norbert Postal Station, Winnipeg, Manitoba, Canada R3V 1L6. (A review of the Selected Papers by the present author will appear in 1993 in a special issue of Math. Comp. that will be published in his memory.) 
DHL is survived by his wife Emma, a son Donald and daughter Laura, four grandchildren, two great-grandchildren, and an older sister Helen. His private papers and 23 personal journals, along with an audiotape of his Gibbs Lecture in Denver in 1965 (paper \#119) and a videotape of his lecture on the history of sieves at the History of Computing meeting at Los Alamos in 1974 (paper \#155) and a videotape of his lecture at the Computer Museum in Boston in 1982, are housed at the Bancroft Library on the U. C. campus at Berkeley. Dr. Robin Rider of the Bancroft Library has stated she would appreciate receiving any correspondence that anyone has had with DHL to be included in the permanent collection there.

It is appropriate here to repeat the final sentence of his obituary at the University of California: "He is sorely missed by all who knew him".

\section{References}

[1] D. N. Lehmer, Factor Table for the First Ten Millions, Carnegie Inst. Publ. 105, Washington, D.C., 1909.

[2] -, List of Prime Numbers from 1 to 10006721, Carnegie Inst. Publ. 165, Washington, D.C., 1914

[3] - Factor Stencils, revised and extended by J. D. Elder, Carnegie Inst., Washington, D.C., Sept., 1939, 1-27; Math. Rev. 1, 133.

[4] - Hunting big game in the theory of numbers, Scripta Math. 1 (1932-33), 229-235.

[5] A. J. Stephens and H. C. Williams, An open architecture number sieve, in: Lecture Notes of the London Math. Soc. 154 (1990), 38-75.

[6] M. Ward, The intrinsic divisors of Lehmer numbers, Ann. of Math. (2) 62(1955), $230-236$.

\section{The PhD students of D. H. Lehmer}

Note that David Singmaster was also a PhD student of R. S. Lehman while DHL was on leave.

1. Donald Marvin Adelman (1947), Some arithmetic properties of sequences of integers satisfying linear recursion sequences.

2. Henry Ludwig Alder (1947), The existence and nonexistence of certain identities in the theory of partitions.

3. William Haddock Simons (1947), Modular functions of stufe 2.

4. Tom Mike Apostol (1948), A study of Dedekind sums and their generalizations.

5. Donald Dines Wall (1949), Normal numbers.

6. Mark Brimhall Wells (1961), Simplification of normal form expressions for Boolean functions of many variables.

7. Nand Kishore (1961), Arithmetical properties of Bessel functions.

8. Jonathan David Young (1962), Application of linear programming to the numerical solutions of linear differential equations.

9. Ronald Lewis Graham (1962), On finite sums of rational numbers. 
10. Robert Samuel Spira (1962), Sums of two squares and Brahmagupta's formula.

11. Jayanthi Chidambaraswamy (1964), Divisibility properties of certain factorials.

12. Harold Mead Stark (1964), On the tenth complex quadratic field with class-number one.

13. Alan Zame (1965), On the distribution of the fractional parts of certain sequences.

14. David Singmaster (1966), On means of differences of consecutive integers relatively prime to $\mathrm{m}$.

15. John David Brillhart (1967), On the Euler and Bernoulli polynomials.

16. David Friedman (1967), Cubic character sums and congruences.

17. James Brown Herreschoff (1968), A theorem on character sums.

18. Richard Paul Stauduhar (1969), The automatic determination of Galois groups.

19. Peter Jay Weinberger (1969), Proof of a conjecture of Gauss on class number two.

DEPARTMENT OF MATHEMATICS

UNIVERSITY OF ARIZONA

TUSCON, ARIZONA 85721

U.S.A.

Received on 17.3.1992

and in revised form on 23.6.1992 


\section{Publications of D. H. Lehmer}

The following list was compiled by DHL. It contains only those publications he considered to be most important. His minor notes that are scattered throughout the literature are not listed.

1. On the use of the calculating machine for cube and fifth roots, Amer. Math. Monthly 32 (1925), 377-379.

2. A cross-division process and its application to the extraction of roots, ibid. 33 (1926), 198-206.

3. On the value of the Napierian base, Amer. J. Math. 48 (1926), 139-143.

4. On the indeterminate equation $t^{2}-p^{2} D u^{2}=1$, Ann. of Math. 27 (1926), 471-476.

5. A list of errors in tables of the Pell equation, Bull. Amer. Math. Soc. 32 (1926), $545-550$.

6. Note on the Mersenne number $2^{139}-1$, ibid., 522 .

7. Tests for primality by the converse of Fermat's theorem, ibid. 33 (1927), 327-340

8. A further note on the converse of Fermat's theorem, ibid. 34 (1928), 54-56.

9. The mechanical combination of linear forms, Amer. Math. Monthly 35 (1928), $114-121$.

10. On the multiple solutions of the Pell equation, Ann. of Math. 30 (1928), 66-72.

11. On Stern's diatomic series, Amer. Math. Monthly 36 (1929), 59-67.

12. On the number $\left(10^{23}-1\right) / 9$, Bull. Amer. Math. Soc. 35 (1929), 349-350.

13. Errors in Kraitchik's table of linear forms, ibid., 865-867.

14. Gauss' third proof of the law of quadratic reciprocity, in: D. E. Smith, A Source Book in Mathematics, 1929, 112-118.

15. On the $r$-th divisors of a number, Amer. J. Math. 52 (1930), 293-304.

16. An extended theory of Lucas' functions, Ann. of Math. 31 (1930), 419-448. $847-850$.

17. A fallacious principle in the theory of numbers, Bull. Amer. Math. Soc. 36 (1930),

18. The p-dimensional analogue of Smith's determinant, Amer. Math. Monthly 37 (1930), 294-296.

19. Sur le nombre $2^{257}-1$, Sphinx 1 (1931), 31-32.

20. On the factorization of Lucas' functions, Tôhoku Math. J. 34 (1931), 1-7.

21. A new calculus of numerical functions, Amer. J. Math. 53 (1931), 843-854.

22. Sur le nombre $2^{149}-1$, Sphinx 1 (1931), 163-165.

23. Arithmetic of double series, Trans. Amer. Math. Soc. 33 (1931), 945-957.

24. On a theorem of von Sterneck, Bull. Amer. Math. Soc. 37 (1931), 723-726.

25. (with R. E. Powers) On factoring large numbers, ibid., 770-776.

26. Arithmetical periodicities of Bessel functions, Ann. of Math. 43 (1932), 143-150.

27. Note on Mersenne numbers, Bull. Amer. Math. Soc. 38 (1932), 383-384.

28. A ternary analogue of Abelian groups, Amer. J. Math. 54 (1932), 329-338.

29. Quasi-cyclotomic polynomials, Amer. Math. Monthly 39 (1932), 383-389. 
30. An inversive algorithm, Bull. Amer. Math. Soc. 38 (1932), 693-694.

31. A note on Fermat's last theorem, ibid., 723-724.

32. On Euler's totient function, ibid., 745-751.

33. Some new factorizations of $2^{n} \pm 1$, ibid. 39 (1933), 105-108.

34. Factorization of certain cyclotomic functions, Ann. of Math. 34 (1933), 461-479.

35. A photo-electric number sieve, Amer. Math. Monthly 40 (1933), 401-406.

36. Numerical notations and their influence on mathematics, Math. News Letter 7 (1933), 5-12. 667.

37. A machine for combining sets of linear congruences, Math. Ann. 109 (1934), 661-

38. On Lucas' test for the primality of Mersenne's numbers, J. London Math. Soc. 10 (1935), 162-165.

39. Lacunary recurrence formulas for the numbers of Bernoulli and Euler, Ann. of Math. 36 (1935), 637-649.

40. On the converse of Fermat's theorem, Amer. Math. Monthly 43 (1936), 347-354.

41. On a conjecture of Ramanujan, J. London Math. Soc. 11 (1936), 114-118.

42. Polynomials for the $n$-ary composition of numerical functions, Amer. J. Math. 58 (1936), 563-572.

43. An extension of the table of Bernoulli numbers, Duke Math. J. 2 (1936), 460-464.

44. A generalized inversive algorithm, Bull. Amer. Math. Soc. 42 (1936), 693-695.

45. On the function $x^{2}+x+A$, Sphinx 6 (1936), 212-214.

46. On the Hardy-Ramanujan series for the partition function, J. London Math. Soc. 12 (1937), 171-176.

47. On the series for the partition function, Trans. Amer. Math. Soc. 43 (1938), 271295.

48. An application of Schläfli's modular equation to a conjecture of Ramanujan, Bull. Amer. Math. Soc. 44 (1938), 84-90.

49. Euclid's algorithm for large numbers, Amer. Math. Monthly 45 (1938), 227-233.

50. Sur les essais directs de primalité, Sphinx 8 (1938), 87-88.

51. A cotangent analogue of continued fractions, Duke Math. J. 4 (1938), 323-340.

52. Sur les nombres $\pi$ and e, Sphinx 8 (1938), 185-187.

53. On arccotangent relations for $\pi$, Amer. Math. Monthly 45 (1938), 657-664.

54. A factorization theorem applied to a test for primality, Bull. Amer. Math. Soc. 45 (1939), 132-137.

55. Note on an absolute constant of Khintchine, Amer. Math. Monthly 46 (1939), $148-152$.

56. On the remainders and convergence of the series for the partition function, Trans. Amer. Math. Soc. 46 (1939), 362-373.

57. On the maxima and minima of Bernoulli polynomials, Amer. Math. Monthly 47 (1940), 533-538.

58. The lattice points of an n-dimensional tetrahedron, Duke Math. J. 7 (1940), 341353.

59. (with Emma Lehmer) On the first case of Fermat's last theorem, Bull. Amer. Math. Soc. 47 (1941), 139-142. 246 .

60. A note on the linear diophantine equation, Amer. Math. Monthly 48 (1941), 240-

61. Guide to Tables in the Theory of Numbers, National Research Council, 1941, 177 pages.

62. Properties of the coefficients of the modular invariant $J(\tau)$, Amer. J. Math. 64 (1942), 488-502. 
63. Recurrence formulas for certain divisor functions, Bull. Amer. Math. Soc. 49 (1943), 150-156.

64. Ramanujan's function $\tau(n)$, Duke Math. J. 10 (1943), 483-492.

65. Note on the computation of the Bessel function $I_{n}(x)$, MTAC 1 (1944), 133-135.

66. The Graeffe process as applied to power series, ibid. 1 (1945), 377-383.

67. Two nonexistence theorems on partitions, Bull. Amer. Math. Soc. 52 (1946), $538-544$.

68. On the factors of $2^{n} \pm 1$, ibid. 53 (1947), 164-167.

69. The vanishing of Ramanujan's function $\tau(n)$, Duke Math. J. 14 (1947), 429-433.

70. The Tarry-Escott problem, Scripta Math. 13 (1947), 37-41.

71. On the partition of numbers into squares, Amer. Math. Monthly 55 (1948), 476-481.

72. A conjecture of Krishnaswami, Bull. Amer. Math. Soc. 54 (1948), 1185-1190.

73. On the converse of Fermat's theorem II, Amer. Math. Monthly 56 (1949), 300-309.

74. Approximations to the area of an $n$-dimensional ellipsoid, Canad. J. Math. 2 (1950), 267-282.

75. Problems concerning Ramanujan's function, in: Proc. Internat. Congress Math. 1, $1950,297$.

76. A triangular number formula for the partition function, Scripta Math. 17 (1951), $17-19$.

77. Ramanujan's function with respect to the modulus 49, Proc. Nat. Inst. Sci. India 17 (1951), 67-70.

78. Mathematical methods in large-scale computing units, in: Proc. Second Sympos. on Large Scale Digital Calculating Machinery, Harvard Univ. Press, Cambridge 1951, $141-146$.

79. The sieve problem for all-purpose computers, MTAC 7 (1953), 6-14.

80. (with Emma Lehmer and H. S. Vandiver) An application of high-speed computing to Fermat's last theorem, Proc. Nat. Acad. Sci. U.S.A. 40 (1954), 25-33.

81. A sieve problem on "pseudo-squares", MTAC 8 (1954), 241-242.

82. The distribution of totatives, Canad. J. Math. 7 (1955), 347-357.

83. On the roots of the Riemann zeta-function, Acta Math. 95 (1956), 291-298.

84. On the diophantine equation $x^{3}+y^{3}+z^{3}=1$, J. London Math. Soc. 31 (1956), $275-280$.

85. High speed computing devices and their applications, in: Modern Mathematics for the Engineer, Chap. 19, McGraw-Hill, New York 1956.

86. On certain character matrices, Pacific J. Math. 6 (1956), 491-499.

87. Extended computation of the Riemann zeta-function, Mathematika 3 (1956), $102-108$.

88. Sorting cards with respect to a modulus, J. Assoc. Comput. Mach. 4 (1957), 41-46.

89. A low energy proof of the reciprocity law, Amer. Math. Monthly 64 (1957), 103106.

90. Discrete variable methods in numerical analysis, in: Proc. Internat. Congress of Mathematicians, 1958, 545-552.

91. On the exact number of primes less than a given limit, Illinois J. Math. 3 (1959), $381-388$.

92. Combinatorial problems with digital computers, in: Proc. Canad. Math. Congress, 1959, 160-173.

93. On a problem of Hardy and Littlewood (with Addendum), J. London Math. Soc. 34 (1959), 395-396, 485.

94. Some functions of Ramanujan, Math. Student 27 (1959), 105-116. 
95. Teaching combinatorial tricks to a computer, in: Proc. Sympos. on Combinatorial Design, USAF Washington 1959, Proc. Sympos. Appl. Math. 10, Amer. Math. Soc., 1960, 179-193.

96. (with Emma Lehmer) On the cubes of Kloosterman sums, Acta Arith. 6 (1960), $15-22$.

97. (with S. Selberg) A sum involving the function of Möbius, ibid., 111-114.

98. Power character matrices, Pacific J. Math. 10 (1960), 895-907.

99. Mahler's matrices, J. Austral. Math. Soc. 1 (1960), Part 4, 385-395.

100. A machine method for solving polynomial equations, J. Assoc. Comput. Mach. 8 (1961), 151-162.

101. (with Emma Lehmer) On runs of residues, Proc. Amer. Math. Soc. 13 (1962), $102-106$.

102. (with Emma Lehmer, W. H. Mills, and J. L. Selfridge) Machine proof of a theorem on cubic residues, Math. Comp. 16 (1962), 407-415.

103. (with Emma Lehmer) Heuristics, anyone?, in: Studies in Mathematical Analysis and Related Topics, Stanford Univ. Press, 1962, 202-210.

104. A note on primitive roots, Scripta Math. 26 (1963), 117-119.

105. (with Emma Lehmer and W. H. Mills) Pairs of consecutive power residues, Canad. J. Math. 15 (1963), 172-177.

106. The complete root-squaring method, J. SIAM 11 (1963), 705-717.

107. Some high-speed logic, in: Proc. Sympos. Appl. Math. 15, Amer. Math. Soc., 1963, 141-145.

108. Automation and pure mathematics, in: Applications of Digital Computers, Ginn and Co., 1963, 219-231.

109. Combinatorial types in number theory calculations, in: Proc. IBM Scientific Computing Sympos. on Combinatorial Problems, Yorktown Heights 1964, 23-30.

110. On a problem of Stфrmer, Illinois J. Math. 8 (1964), 57-79.

111. Number systems, in: McGraw-Hill Encyclopedia of Science and Technology, 1964, $257-260$.

112. (with J. Brillhart and Emma Lehmer) Bounds for pairs of consecutive seventh and higher power residues, Math. Comp. 18 (1964), 397-407.

113. The machine tools of combinatorics, in: Applied Combinatorial Mathematics, Wiley, 1964, 5-31.

114. The primality of Ramanujan's tau-function, Amer. Math. Monthly 72 (1965), No. 2, Part II, 15-18.

115. The prime factors of consecutive integers, ibid., 19-20.

116. Permutation by adjacent interchanges, ibid., 36-46.

117. On certain chains of primes, Proc. London Math. Soc. (3) 14A (1965), 183-186.

118. Some properties of the cyclotomic polynomial, J. Math. Anal. Appl. 15 (1966), $105-117$.

119. Mechanized mathematics, Bull. Amer. Math. Soc. 72 (1966), 739-750; Polish transl.: Wiadom. Mat. 10 (1968), 183-194.

120. (with Emma Lehmer) The cyclotomy of Kloosterman sums, Acta Arith. 12 (1967), 385-407.

121. (with Emma Lehmer) The cyclotomy of hyper-Kloosterman sums, ibid. 14 (1968), 89-111.

122. Machines and pure mathematics, in: Computers in Mathematical Research, North-Holland, Amsterdam 1968, 1-7.

123. Search procedures for polynomial equation solving, in: Constructive Aspects of the Fundamental Theorem of Algebra, Wiley-Interscience, New York 1969, 193-208. 
124. Computer technology applied to the theory of numbers, in: Studies in Number Theory, MAA Stud. Math. 6, 1969, 117-151.

125. (with Emma Lehmer and D. Shanks) Integer sequences having prescribed quadratic character, Math. Comp. 24 (1970), 433-451.

126. Note on the distribution of Ramanujan's tau function, ibid., 741-743.

127. The economics of number theoretic computation, in: Computers in Number Theory, Proc. Atlas Sympos. No. 2, Oxford 1969, Academic Press, New York 1971, 1-9.

128. Permutations with strongly restricted displacements, in: Combinatorial Theory and Its Applications (Balatonfüred, 1969), II, Colloq. Math. Soc. János Bolyai 4, NorthHolland, Amsterdam 1970, 755-769.

129. Effective enumeration methods for highly restricted permutations, in: Proc. Canad. Math. Congress, 1971, 103-116.

130. On the compounding of certain means, J. Math. Anal. Appl. 36 (1971), 183-200.

131. Some recursive sequences, in: Proc. Manitoba Conf. on Numerical Math., Winnipeg, 1971, 15-30.

132. On reciprocally weighted partitions, Acta Arith. 21 (1972), 379-388.

133. Calculating moments of partitions, in: Proc. Second Manitoba Conf. on Numerical Math., Winnipeg, 1972, 217-220.

134. Continued fractions containing arithmetic progressions, Scripta Math. 29 (1973), $17-24$.

135. Some properties of circulants, J. Number Theory 5 (1973), 43-54.

136. Multisectioned moments of Stirling numbers of the second kind, J. Combin. Theory Ser. A 15 (1973), 210-224.

137. (with R. K. Guy, J. L. Selfridge, and M. C. Wunderlich) Second report on aliquot series, in: Proc. Third Manitoba Conf. on Numerical Math., 1973, 357-368.

138. (with Emma Lehmer) A new factorization technique using quadratic forms, Math. Comp. 28 (1974), 625-635.

139. The influence of computing on research in number theory, in: Proc. Sympos. Appl. Math. 20, Amer. Math. Soc., 1974, 3-12.

140. Combinatorial and cyclotomic properties of certain tridiagonal matrices, in: Proc. Fifth Southeastern Conf. on Combinatorics, Graph Theory, and Computing, 1974, 53-74.

141. Fibonacci and related sequences in periodic tridiagonal matrices, Fibonacci Quart. 13 (1975), 150-158.

142. Euler constants for arithmetical progressions, Acta Arith. 27 (1975), 125-142.

143. (with J. D. Brillhart and J. L. Selfridge) New primality criteria and factorizations of $2^{m} \pm 1$, Math. Comp. 29 (1975), 620-647.

144. Harry Schultz Vandiver, 1882-1973, Bull. Amer. Math. Soc. 80 (1974), 817-818.

145. The characters of linear permutations, Linear and Multilinear Algebra 4 (1976), $1-16$.

146. Strong Carmichael numbers, J. Austral. Math. Soc. 21 (1976), 508-510.

147. (with R. L. Graham) On the permanent of Schur's matrix, ibid., 487-497.

148. Incomplete Gauss sums, Mathematika 23 (1976), 125-135.

149. Exploitation of parallelism in number theoretic and combinatorial computations, in: Proc. Sixth Manitoba Conf. on Numerical Math. 1976, Utilitas Math. Publ., Winnipeg, 95-111.

150. Prime factors of cyclotomic class numbers, Math. Comp. 31 (1977), 599-607.

151. (with J. M. Masley) Table of the cyclotomic class numbers $h^{*}(p)$ and their factors for $200<p<521$, ibid. 32 (1978), 577-582.

152. Permutations, partitions, permanents, parallelism, in: AFCET-SMF, First Colloquium, Palaiseau, France, 1 (1978), 123-137. 
153. (with Emma Lehmer) Picturesque exponential sums, I, Amer. Math. Monthly 86 (1979), 725-733.

154. On a constant of Turán and Erdös, Acta Arith. 37 (1980), 359-361.

155. A history of the sieve process, in: A History of Computing in the Twentieth Century, Academic Press, New York 1980, 445-456.

156. (with Emma Lehmer) Picturesque exponential sums. II, J. Reine Angew. Math. 318 (1980), 1-19.

157. (with Emma Lehmer) Cyclotomy for non-squarefree moduli, in: Lecture Notes in Math. 899, Springer, 1980, 276-300.

158. On Fermat's quotient, base two, Math. Comp. 36 (1981), 289-290.

159. Remarks on 01 matrices, Congr. Numer. 30 (1981), 29-44.

160. Generalized Eulerian numbers, J. Combin. Theory Ser. A 32 (1982), 195-215.

161. (with Emma Lehmer) Multiple sums of cyclotomic numbers, Utilitas Math. 23 (1983), 223-239.

162. Computational advantages of cyclotomic fields, Congr. Numer. 38 (1983), 133-137.

163. (with Emma Lehmer) Properties of polynomials having Fibonacci numbers for coefficients, Fibonacci Quart. 21 (1983), 62-64.

164. (with Emma Lehmer) Cyclotomy with short periods, Math. Comp. 41 (1983), $743-758$.

165. (with J. Brillhart, J. L. Selfridge, B. Tuckerman, and S. Wagstaff, Jr.), Factorizations of $b^{n} \pm 1, b=2,3,5,6,7,10,11,12$, up to high powers, Contemp. Math. 22, Amer. Math. Soc., 1983, 2nd ed. 1988.

166. (with Emma Lehmer) The sextic period polynomial, Pacific J. Math. 111 (1984), $341-355$.

167. The chromatic polynomial of a graph, ibid. 118 (1985), 463-469.

168. Numbers associated with Stirling numbers and $x^{x}$, Rocky Mountain J. Math. 15 (1985), 461-479.

169. Interesting series involving the central binomial coefficient, Amer. Math. Monthly 92 (1985), 449-457.

170. Coloring the Platonic solids, ibid. 93 (1986), 288-292.

171. (with K. Mahler and A. J. van der Poorten) Integers with digits 0 and 1 , Math. Comp. 46 (1986), 683-689.

172. (with Emma Lehmer) Cyclotomic resultants, ibid. 48 (1987), 211-216.

173. The sum of like powers of the zeros of the Riemann zeta function, ibid. 50 (1988), 265-273.

174. A half century of reviewing, in: A Century of Mathematics in America, I, Amer. Math. Soc., 1988, 265-266.

175. A new approach to Bernoulli polynomials, Amer. Math. Monthly 95 (1988), 905911.

176. A matrix paraphrase of cyclotomy, Acta Arith. 53 (1990), 357-365.

177. Factorization then and now, in: Computing in Mathematics, Lecture Notes in Pure Appl. Math. 125, Marcel Dekker, New York 1990, 311-320.

178. A matrix paraphrase of Kloosterman sums, Acta Arith. 56 (1990), 83-92.

179. Matrix paraphrases, Linear and Multilinear Algebra 28 (1991), 251-264.

180. The number of solutions of a certain congruence involving the sum of like powers, Utilitas Math. 39 (1991), 65-89.

181. (with Emma Lehmer) The Lehmer project, Math. Comp., to appear. 